\title{
Torsade de pointes tachycardia as a rare manifestation of acute enteroviral myocarditis
}

\author{
C Badorff, A M Zeiher, S H Hohnloser
}

\begin{abstract}
A patient with cardiac arrest and documented torsade de pointes ventricular tachycardia is presented in whom acute coxsackievirus B2 myocarditis was identified as the most likely underlying cardiac condition. This case shows that torsade de pointes may occur as a rare manifestation of viral myocarditis. Serial serological tests and endomyocardial biopsies may be helpful in establishing a diagnosis in such patients.

(Heart 2001;86:489-490)
\end{abstract}

Keywords: torsade de pointes; ventricular tachycardia; viral myocarditis

Torsade de pointes is a polymorphous ventricular tachycardia in which the QRS complexes oscillate around the isoelectrical line. It occurs in the presence of a hereditary or acquired long QT syndrome, the latter being most commonly induced by drugs or toxins. ${ }^{12}$ However, only sparse reports exist indicating an association of acute myocarditis with this form of arrhythmia. ${ }^{34}$

Case report

A 39 year old female patient was successfully resuscitated from out of hospital cardiac arrest caused by ventricular fibrillation. Her medical history was unremarkable apart from a flu like illness two weeks previously. Since the 12 lead

Accepted 2 July 2001
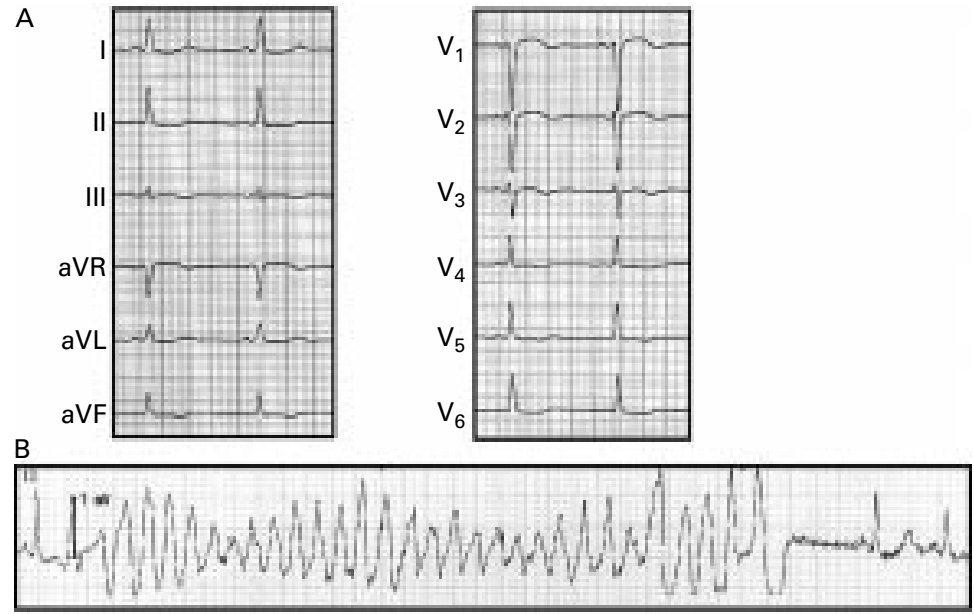

Figure 1 (A) Initial 12 lead ECG $(25 \mathrm{~mm} / \mathrm{s})$ with anterior $T$ wave inversions. (B) Torsade de pointes like ventricular tachycardia. The sinus beat following spontaneous termination of the tachycardia shows a prolonged $Q T$ duration.
ECG showed anterior $\mathrm{T}$ wave inversion (fig $1 \mathrm{~A})$ and both creatine kinase $(258 \mathrm{U} / \mathrm{l})$ and creatine kinase $\mathrm{MB}$ fraction (36 U/1) were increased, acute myocardial infarction was initially suspected. However, on angiography the coronary arteries were seen to be free of significant stenoses. Importantly, left ventricular function was significantly impaired (ejection fraction $25-30 \%$ ) with an enlarged left ventricular diastolic diameter $(65 \mathrm{~mm})$ and an increased left ventricular end diastolic pressure (20 mm Hg), consistent with a dilated cardiomyopathy phenotype.

Subsequently, the patient developed nonsustained, torsade de pointes ventricular tachycardia in the setting of a prolonged QT duration (fig 1B). Serum electrolytes were within normal limits and the patient was not receiving any QT prolonging drugs. Most likely, the previous episode of ventricular fibrillation had been caused by secondary degeneration of a similar torsade de pointes.

On the basis of the medical history acute myocarditis was suspected. Therefore, a first serum sample was drawn for determination of viral titres and endomyocardial biopsies were taken from the right side of the interventricular septum.

Routine haematoxylin and eosin staining showed an increased interstitial area between the cardiomyocytes with oedema, fibrosis, and enhanced cellularity (fig 2A). Immunostaining for the common leucocyte antigen (CD45) identified multiple inflammatory infiltrates in the myocardium (fig 2B). Since enteroviruses are common aetiological agents in viral myocarditis, immunostaining was performed using an antibody against the enterovirus group specific capsid antigen VP-1 as described. ${ }^{5}$ An area encompassing several virally infected cardiomyocytes with a preferential sarcolemmal VP-1 signal was detected (fig 2C). The same sarcolemmal staining pattern for VP-1 was observed in mouse hearts experimentally infected with coxsackievirus B3 (fig 2D). Control stains with an isotype control mouse IgG as primary antibody did not produce any signal (data not shown).

A second serum sample taken from the patient 10 days later showed a fourfold increase (from $1: 40$ to $1: 160$ ) of the neutralising antibody titre against coxsackievirus B2. Titres against all other coxsackievirus serotypes tested (A9, A21, B1, B3-6), parvovirus B19, herpes 

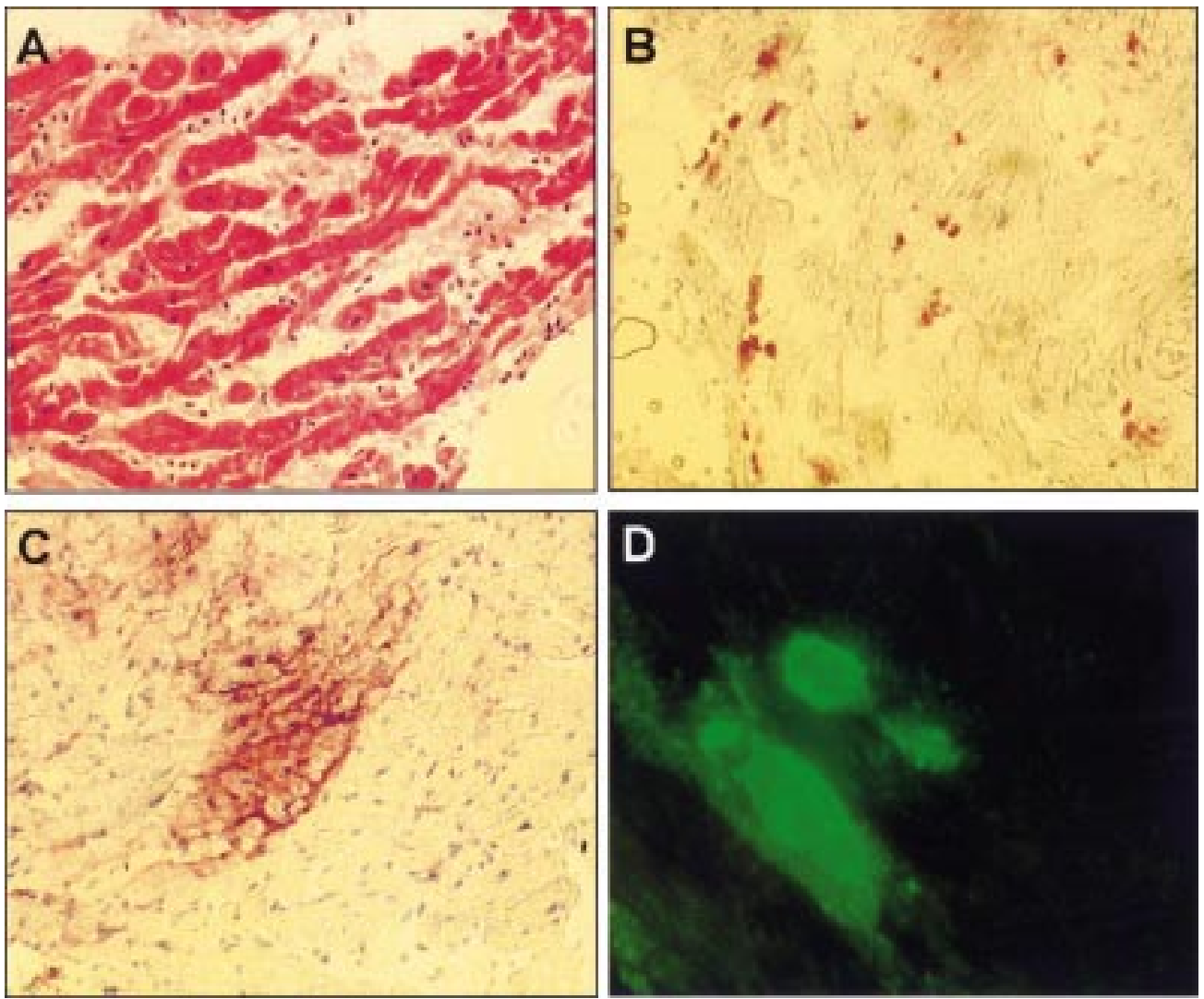

Figure 2 Endomyocardial biopsy results. (A) Haematoxylin and eosin staining, $\times 200$. (B) CD45 immunostaining with horseradish peroxidase signal detection, $\times 200$. Note the abundance and foci of inflammatory cells. $(C)$ Immunostaining for the enteroviral VP-1 antigen with horseradish peroxidase signal detection, $\times 200$. Note the strong sarcolemmal staining pattern in a group of cardiomyocytes. (D) VP-1 immunofluorescence staining of a mouse heart experimentally infected with coxsackievirus B3, $\times 400$. The virally infected cells show a strong signal similar to the pattern described in $C$.

simplex virus, cytomegalovirus, and adenovirus remained constant.

Taken together, these findings identified acute coxsackievirus B2 myocarditis as the most likely cause of the torsade de pontes ventricular tachycardia in our patient.

\section{Discussion}

Our patient illustrates three clinical presentations of viral myocarditis: acute myocardial infarction, recent onset dilated cardiomyopathy, and sudden death. ${ }^{6}$ In principal, any arrhythmia may complicate viral myocarditis where the lytic nature of the viral infection or the resulting inflammatory reaction may cause inhomogeneous repolarisation and an unstable membrane potential in affected cardiomyocytes. These abnormalities may facilitate ventricular tachyarrhythmias through re-entrant mechanisms or enhanced autonomy, respectively. We previously reported a mechanism that may underlie these effects: cleavage of the cytoskeletal protein dystrophin by the coxsackieviral protease $2 \mathrm{~A}$ with subsequent disruption of the membrane-anchoring dystrophin glycoprotein complex and enhanced sarcolemmal permeability in vitro and in vivo. ${ }^{7}$
In summary, torsade de pointes ventricular tachycardia can occur as a rare but life threatening manifestation of acute viral myocarditis. Viral myocarditis may be considered as a potential cause in aetiologically unclear cases of torsade de pointes. Serological tests as well as endomyocardial biopsies may be helpful in establishing a diagnosis in these patients.

Supported by a grant from the Deutsche Forschungsgemeinschaft to CB (Ba 1668/3-1).

1 Dessertenne F. La tachycardie ventriculaire a deux foyers opposes variable. Arch Mal Coeur 1966;59:263-72.

2 El-Sherif N, Turitto G. The long QT syndrome and torsade de pointes. PACE 1999;22:91-110.

3 Sareli P, Schamroth CL, Passias J, et al. Torsade de pointes due to coxsackie B3 myocarditis. Clin Cardiol 1987;10:3612.

4 Fiddler GI, Campbell RWF, Pottage A, et al. Varicella myocarditis presenting with unsual ventricular arrhythmias. $\mathrm{Br}$ Heart f 1977;39:1150-3.

5 Li Y, Bourlet T, Andreoletti L, et al. Enteroviral capsid protein VP1 is present in myocardial tissues from some patients with myocarditis or dilated cardiomyopathy. Circulation 2000;101:231-4.

6 Karjalainen J, Heikkila J. Incidence of the three presentations of acute myocarditis in young men in military service: a 20-year experience. Eur Heart F 1999;20:1120-5.

7 Badorff C, Lee GH, Lamphear BJ, et al. Enteroviral protease 2A cleaves dystrophin: evidence of cytoskeletal disruption in an acquired cardiomyopathy. Nat Med 1999;5:320-6. 